\title{
IMPLEMENTAÇÃO COMPUTACIONAL DO MODELO CARGA-FLUXO DE CARGA-FLUXO DE DIPOLO PARA CÁLCULO E INTERPRETAÇÃO DAS INTENSIDADES DO ESPECTRO INFRAVERMELHO
}

\author{
Thiago C. F. Gomes, João Viçozo da Silva Jr., Luciano N. Vidal*, Pedro A. M. Vazquez e Roy E. Bruns \\ Departamento de Físico-Química, Instituto de Química, Universidade Estadual de Campinas, CP 6154, 13084-862 Campinas - SP, \\ Brasil
}

Recebido em 25/3/08; aceito em 6/5/08; publicado na web em 22/9/08

\begin{abstract}
COMPUTATIONAL IMPLEMENTATION OF THE MODEL CHARGE-CHARGE FLUX-DIPOLE FLUX FOR CALCULATION AND ANALYSIS OF INFRARED INTENSITIES. The first computational implementation that automates the procedures involved in the calculation of infrared intensities using the charge-charge flux-dipole flux model is presented. The atomic charges and dipoles from the Quantum Theory of Atoms in Molecules (QTAIM) model was programmed for Morphy98, Gaussian98 and Gaussian03 programs outputs, but for the ChelpG parameters only the Gaussian programs are supported. Results of illustrative but new calculations for the water, ammonia and methane molecules at the MP2/6-311++G(3d,3p) theoretical level, using the ChelpG and QTAIM/Morphy charges and dipoles are presented. These results showed excellent agreement with analytical results obtained directly at the MP2/6$311++\mathrm{G}(3 \mathrm{~d}, 3 \mathrm{p})$ level of theory.
\end{abstract}

Keywords: $a b$ initio and DFT electronic structure methods; CCFDF/QTAIM and CCFDF/ChelpG absolute infrared intensities; molecular spectroscopy.

\section{INTRODUÇÃO}

A absorção de radiação eletromagnética na região do infravermelho é comumente descrita por modelos semiclássicos, onde o campo elétrico obedece às equações do eletromagnetismo clássico e o sistema interagente é modelado pela mecânica quântica. No processo de derivação da expressão para a intensidade da radiação absorvida, a abordagem utilizada com maior freqüência é a Aproximação Duplamente Harmônica, ${ }^{1}$ onde o campo de força molecular é descrito por um potencial harmônico e a dependência do momento dipolar elétrico nas coordenadas normais de vibração é expandida em série de Taylor até o termo de primeira ordem. Dentro desta aproximação, a intensidade de uma transição fundamental é proporcional ao quadrado da derivada do momento dipolar molecular com relação à coordenada normal de vibração, ${ }^{2}$ ou seja, $A_{k} \alpha\left(\partial \mathbf{p} / \partial Q_{k}\right)^{2}$.

Atualmente, implementações computacionais para o cálculo de intensidades infravermelhas são bastante freqüentes em pacotes de química quântica, onde estas propriedades são obtidas através de métodos ab initio ou através da Teoria do Funcional da Densidade..$^{3-6}$ O procedimento usado para obter as intensidades envolve o cálculo das derivadas geométricas do momento dipolar elétrico, obtendo-se os Tensores Polares Atômicos, seguido pela projeção destes gradientes no sistema de coordenadas normais de vibração e, por fim, a absorbância $A_{k}$ é obtida. Seguindo esta metodologia, as intensidades infravermelhas das transições fundamentais de moléculas em fase gasosa podem ser calculadas com boa exatidão. Por exemplo, em um estudo realizado para doze fluor-cloro-metanos, no nível de teoria MP2/6-311++G(3d,3p), o erro RMS (root-mean-square error) obtido comparando-se valores teóricos com dados experimentais é de $23,1 \mathrm{~km} / \mathrm{mol},{ }^{7}$ um resultado bastante satisfatório, uma vez que as intensidades observadas experimentalmente apresentam um erro médio de cerca de $8,6 \mathrm{~km} / \mathrm{mol}$.

Apesar do formalismo empregado no cálculo das intensidades infravermelhas prever com boa precisão estas intensidades, a descrição

*e-mail: lnvidal@iqm.unicamp.br fenomenológica da absorção da radiação infravermelha restringe-se à análise dos Tensores Polares Atômicos e das absorbâncias $A_{k}$. Visando estender a descrição deste fenômeno, novas metodologias de análise foram desenvolvidas. A idéia central destes modelos está no particionamento da derivada do momento dipolar elétrico molecular em diversas contribuições atômicas, tais como, cargas estáticas, fluxos de carga e termos de sobreposição. ${ }^{8-10}$ Os modelos propondo o particionamento destas derivadas empregando cargas atômicas de Mulliken ${ }^{11-13}$ ou GAPT (Generalized Atomic Polar Tensor) $)^{14}$ e termos de fluxo de carga atômica, obtidos através de derivadas geométricas destas cargas, carecem de uma interpretação física adequada. Isso foi contornado através da inclusão no modelo de mais um termo da expansão multipolar do dipolo elétrico molecular $\mathbf{p}$, que são os dipolos atômicos $\mathbf{p}^{(\alpha)}$ : 15

$p_{\sigma} \approx \sum_{\alpha=1}^{N} q^{(\alpha)} \sigma_{\alpha}+\sum_{\alpha=1}^{N} p_{\sigma}^{(\alpha)}$

$\sigma=x, y$ ou $z ; q^{(\alpha)}=$ carga do $\alpha$-ésimo átomo; $\mathrm{N}=$ número de átomos na molécula

A inclusão dos dipolos atômicos na descrição do dipolo molecular leva a um novo esquema de decomposição das derivadas do dipolo molecular, onde, além das componentes de carga e fluxo de cargas atômicas, surge uma componente de fluxo de dipolo atômico. Este tipo de decomposição dos Tensores Polares Atômicos, conhecida pelo acrônimo em inglês CCFDF ${ }^{16,17}$ (Charge-Charge Flux-Dipole Flux), possibilita uma nova interpretação das mudanças ocorridas na estrutura eletrônica durante os movimentos de vibração que é bastante atrativa porque utiliza somente conceitos físicos clássicos de simples interpretação. Na teoria CCFDF, a intensidade de uma transição fundamental tem origem em três processos fisicamente distintos: a presença de cargas atômicas estáticas na direção do movimento de vibração (termo de carga); a transferência intramolecular de carga ocorrida durante a vibração (fluxo de carga) e, a polarização da densidade eletrônica em 
virtude da deformação molecular decorrente do movimento vibratório, resultando em uma variação dos momentos dipolares atômicos (fluxo de dipolo).

Uma questão inerente ao modelo CCFDF diz respeito ao cálculo das cargas e dos dipolos atômicos. Em princípio, não existem maiores restrições quanto à teoria a ser utilizada para determinar estas quantidades, no entanto, existem qualidades que são altamente desejáveis nestes modelos, tais como, ${ }^{14}$ as cargas atômicas devem ser invariantes às rotações e translações da molécula, a soma das cargas atômicas deve reproduzir a carga total do sistema, as cargas e os dipolos atômicos devem refletir a simetria molecular; estas duas propriedades devem convergir para um valor bem definido, à medida que as funções de base se tornam mais completas, devem possuir uma interpretação física bem definida e a soma dos dipolos atômicos deve reproduzir o momento dipolar elétrico do sistema. Um modelo que detém estas qualidades além de ser fisicamente bastante atrativo, em função da forma como o problema do cálculo de cargas e dipolos atômicos é tratado, é a Teoria Quântica dos Átomos em Moléculas (QTAIM) proposta por Bader. ${ }^{18,19}$ Nesta abordagem, as fronteiras entre os átomos são determinadas através do cálculo das superfícies de fluxo zero, onde o gradiente da densidade eletrônica deixa de variar e as propriedades atômicas são obtidas pela integração da densidade eletrônica no espaço em torno do átomo definido por estas superfícies. Em estudos CCFDF/QTAIM de intensidades infravermelhas no nível de teoria MP2/6-311 ++G(3d,3p), a concordância entre os valores das intensidades derivadas a partir desta metodologia com os valores determinados diretamente de cálculos MP2/6-311++G(3d,3p) é excelente, exibindo erros RMS variando entre $\pm 1,4$ e $\pm 5,6 \mathrm{~km} / \mathrm{mol}^{7,15,17,20}$ Além disso, resultados inovadores foram observados através da análise CCFDF/QTAIM das intensidades infravermelhas, como um elevado coeficiente de correlação negativa (cerca de -0,9) calculado entre as contribuições de fluxo de carga e de fluxo de dipolo, indicando que a transferência de carga de uma região a outra da molécula durante as vibrações é acompanhada por um efeito de relaxação da polarização da densidade eletrônica na direção oposta.

Em virtude do modelo CCFDF requerer a execução de uma série de tarefas bastante complexas, como a análise de coordenadas normais; o cálculo numérico de derivadas de cargas e de dipolos atômicos; a geração de grandezas tensoriais, a sua aplicação é bastante difícil e complexa.

Com a finalidade de automatizar o procedimento de análise CCFDF, este modelo foi implementado no programa Placzek, ${ }^{21,22}$ permitindo que os Tensores Polares Atômicos e sua decomposição em termos de Carga-Fluxo de Carga-Fluxo de Dipolo seja efetuada de maneira totalmente automatizada. $\mathrm{O}$ algoritmo desenvolvido explora a simetria molecular, reduzindo o número de cálculos necessários. A presente implementação permite o uso dos modelos para cálculo de cargas e de dipolos atômicos QTAIM e ChelpG. ${ }^{23,24}$

\section{TEORIA E IMPLEMENTAÇÃO COMPUTACIONAL}

Dentro da Aproximação Duplamente Harmônica,${ }^{1}$ a energia potencial molecular é expressa pelo potencial harmônico e a variação do momento dipolar elétrico total nas coordenadas de deslocamento atômicas é linear, a intensidade de uma transição fundamental no espectro infravermelho é obtida calculando-se o quadrado da norma euclidiana do vetor derivada do momento dipolar elétrico molecular, $\left(\partial \mathrm{p} / \partial Q_{k}\right)$ :

$A_{k}=\frac{N_{A} \pi}{3 c^{2}}\left\{\left\|\left(\frac{\partial \mathbf{p}}{\partial Q_{k}}\right)\right\|_{e q} \|_{2}\right\}^{2}$

$k=1, \ldots, 3 \mathrm{~N}-5$ sistema linear ou $k=1, \ldots, 3 \mathrm{~N}-6$ sistema não linear sendo $Q_{k}$ a k-ésima coordenada normal de vibração, $N_{A}$ o número de Avogadro, $c$ a velocidade da luz no vácuo e $N$ o número de átomos na molécula. Esta derivada é calculada inicialmente no sistema de coordenadas cartesianas atômicas, $\{\xi\}$, e posteriormente projetada no sistema de coordenadas normais, utilizando um vetor unitário ponderado em massa, $\mathbf{L}_{\mathrm{Q}_{\mathrm{k}}}$, que aponta na direção da k-ésima vibração:

$$
\begin{array}{r}
\left(\frac{\partial p_{\sigma}}{\partial Q_{k}}\right)_{e q}=\left(\nabla_{\xi} p_{\sigma}\right) \cdot \mathbf{L}_{Q_{k}}, \quad\left(\nabla_{\xi}\right)_{i}=\frac{\partial}{\partial \xi_{i}} \\
\xi_{i}=1, \ldots, 3 N
\end{array}
$$

Para permitir a interpretação das intensidades infravermelhas em termos de parâmetros atômicos, o formalismo dos Tensores Polares Atômicos, TPA, ${ }^{25,26}$ é aplicado ao gradiente do dipolo molecular, que é então decomposto em parcelas atômicas:

$\mathbf{P}_{X}^{(\alpha)} \equiv \nabla_{\xi}^{(\alpha)} \mathbf{p}, \quad \nabla_{\xi}^{(\alpha)} \equiv\left[\frac{\partial}{\partial x_{\alpha}} ; \frac{\partial}{\partial y_{\alpha}} ; \frac{\partial}{\partial z_{\alpha}}\right], \quad \alpha=1, \ldots, N$

O modelo CCFDF tem início com a redefinição do momento dipolar molecular, p, particionando-o em contribuições de cargas atômicas, $q^{(\alpha)}$, e dipolos atômicos, $\mathbf{p}^{(\alpha)}$ :

$p_{\sigma} \equiv \sum_{\alpha=1}^{N} q^{(\alpha)} \sigma_{\alpha}+\sum_{\alpha=1}^{N} p_{\sigma}^{(\alpha)}$

Quando o operador gradiente atômico $\nabla_{\xi}^{(\alpha)}$ atua sobre este novo momento dipolar, um novo TPA é gerado, composto por três parcelas distintas: ${ }^{16,17}$ o termo de carga atômica $\mathbf{C}_{\mathrm{x}}{ }^{(\alpha)}$, o de fluxo de carga atômica $\mathbf{F C}_{\mathrm{x}}{ }^{(\alpha)}$ e o de fluxo de dipolo atômico $\mathbf{F D}_{\mathrm{x}}{ }^{(\alpha)}$ :

$\nabla_{\xi}{ }^{(\alpha)} \mathbf{p}=\mathbf{C}_{\mathrm{x}}{ }^{(\alpha)}+\mathbf{F} \mathbf{C}_{\mathrm{x}}{ }^{(\alpha)}+\mathbf{F} \mathbf{D}_{\mathrm{x}}{ }^{(\alpha)}$

$\left(\mathbf{C}_{X}^{(\alpha)}\right)_{b \rho}=\left\{\begin{array}{l}q^{(\alpha)}, \text { se } \sigma=\rho \\ 0, \text { se } \sigma \neq \rho\end{array}\right.$

$\left(\mathbf{F C}_{X}^{(\alpha)}\right)_{b \rho}=\sum_{\beta=1}^{N} \sigma_{\beta}\left(\frac{\partial q^{(\beta)}}{\partial \rho_{\alpha}}\right)_{e q}$

$\left.\mathbf{F D}_{X}^{(\alpha)}\right)_{b \rho}=\sum_{\beta=1}^{N}\left(\frac{\partial p_{\sigma}^{(\beta)}}{\partial \rho_{\alpha}}\right)_{e q}, \quad \sigma, \rho=x, y$ ou $z$

$\mathrm{Na}$ etapa final para obter as intensidades infravermelhas no modelo CCFDF, é feita uma mudança de base, passando este novo conjunto de tensores polares para o sistema de coordenadas normais. Inicialmente, compõem-se matrizes de dimensão $3 \times 3 N$, denominadas Tensor Polar Molecular de Carga $\mathbf{C}_{\mathrm{x}}$, de Fluxo de Carga $\mathbf{F} \mathbf{C}_{\mathrm{x}}$ e de Fluxo de Dipolo $\mathbf{F D}_{\mathrm{x}}$, formadas através da justaposição dos respectivos TPA:

$\mathbf{C}_{\mathrm{x}} \equiv\left\{\mathbf{C}_{\mathrm{x}}^{(1)} \mathbf{C}_{\mathrm{x}}^{(2)} \cdots \mathbf{C}_{\mathrm{x}}{ }^{(\mathrm{N})}\right\}$

$\mathbf{F C}_{\mathrm{x}} \equiv\left\{\mathbf{F C}_{\mathrm{x}}^{(1)} \mathbf{F C}_{\mathrm{x}}{ }^{(2)} \cdots \mathbf{F C}_{\mathrm{x}}{ }^{(\mathrm{N})}\right\}$

$\mathbf{F D}_{\mathrm{x}} \equiv\left\{\mathbf{F D}_{\mathrm{x}}^{(1)} \mathbf{F D}_{\mathrm{x}}^{(2) \ldots .} \mathbf{F D}_{\mathrm{x}}^{(\mathrm{N})}\right\}$

$\mathbf{P}_{\mathrm{x}} \equiv \mathbf{C}_{\mathrm{x}}+\mathbf{F C}_{\mathrm{x}}+\mathbf{F D}$ 
Na etapa seguinte, a derivada do vetor momento dipolar molecular é obtida através do produto de matrizes

$\left(\frac{\partial \mathbf{p}}{\partial Q_{k}}\right)_{e q}=\mathbf{P}_{X} \mathbf{L}_{Q_{k}}=\mathbf{C}_{Q_{k}}+\mathbf{F} \mathbf{C}_{Q_{k}}+\mathbf{F} \mathbf{D}_{Q_{k}}$

permitindo que as intensidades CCFDF sejam obtidas pelo uso da Equação 2.

Na implementação do modelo CCFDF efetuada no programa Placzek, as derivadas em torno da geometria de equilíbrio das cargas atômicas, $q^{(\alpha)}$, e das componentes dos dipolos atômicos, $\mathrm{p}_{\sigma}^{(\alpha)}$, no sistema de coordenadas cartesianas (Equações 8 e 9), são obtidas numericamente através de fórmulas de diferenciação central de dois pontos. ${ }^{27}$ Estas cargas e dipolos atômicos, na teoria QTAIM ou ChelpG, são calculadas, respectivamente, através das implementações destes modelos existentes nos programas Morphy $98^{28}$ e Gaussian, ${ }^{3}$ podendo ser obtidas nos níveis de teoria Hartree-Fock, MP2, através de funções de onda multiconfiguracionais e também pela Teoria do Funcional da Densidade (DFT), o que, por conseguinte, permite que a análise CCFDF seja realizada nestes mesmos níveis de teoria. Durante um cálculo de intensidades CCFDF, o programa Placzek executa de forma totalmente automática a seguinte sequiência de ações: geração da malha de pontos necessária ao cálculo das derivadas de $q^{(\alpha)}$ e $\mathbf{p}^{(\alpha)}$, na forma de um conjunto de arquivos contendo a função de onda do sistema que será utilizada pelo programa Morphy98 ou Gaussian; chamada do programa que irá realizar os cálculos das cargas e dos dipolos atômicos; terminados estes cálculos, estas propriedades são lidas nos arquivos de saída; os termos de fluxo de carga e de fluxo dipolo atômico são calculados e a análise CCFDF é realizada obtendose as propriedades apresentadas nas Equações 2-14.

No que tange à análise de coordenadas normais, requerida para determinar as frequiências vibracionais assim como os vetores $\mathbf{L}_{Q_{k}}$ da Equação 3, o programa Placzek pode gerar estas quantidades a partir de campos de força moleculares ab initio, DFT ou empíricos. Detalhes da implementação computacional para este tipo de propriedade estão descritos na ref. 21.

\section{Simetria no cálculo de derivadas}

No caso de moléculas contendo dois ou mais átomos simetricamente equivalentes, ou seja, contendo átomos de mesmo número atômico e massa isotópica cujo vetor posicional de um possa ser transformado no do outro por meio de operações de rotação, o programa Placzek permite que as derivadas associadas a átomos nesta situação sejam calculadas apenas para um deles, sendo as derivadas dos demais geradas através de transformações unitárias. Para descrever como estas derivadas são obtidas, suponhamos que $A$ e $B$ sejam átomos simetricamente equivalentes, que A e B sejam seus vetores de posição e que $\mathbf{R}$ seja uma matriz unitária com a seguinte propriedade: $\mathbf{B}=\mathbf{R} \cdot \mathbf{A}$. Então, $\mathbf{P}_{\mathrm{x}}^{(B)}$ será obtido a partir de $\mathbf{P}_{\mathrm{x}}{ }^{(A)}$ através da transformação: ${ }^{29}$

$\mathbf{P}_{X}^{(B)}=\mathbf{R} \cdot \mathbf{P}_{X}^{(A)} \cdot \widetilde{\mathbf{R}}$

Os tensores polares atômicos $\mathbf{C}_{\mathrm{x}}{ }^{(\mathrm{b})}, \mathbf{F} \mathbf{C}_{\mathrm{x}}{ }^{(\mathrm{b})} \mathrm{e} \mathbf{F D}_{\mathrm{x}}{ }^{(\mathrm{b})}$ são gerados de forma completamente análoga.

\section{DETALHES COMPUTACIONAIS}

Para ilustrar a implementação no programa Placzek do cálculo de intensidades no espectro infravermelho através do modelo de CCFDF, estas propriedades foram obtidas neste trabalho, pela primeira vez, para $\mathrm{H}_{2} \mathrm{O}, \mathrm{NH}_{3}$ e $\mathrm{CH}_{4}$, utilizando os modelos de cargas e dipolos atômicos ChelpG e QTAIM. Com o objetivo de poder comparar os resultados deste trabalho com os resultados publicados pelo grupo nos últimos anos, todas as propriedades apresentadas neste trabalho foram calculadas no nível de teoria MP2/6-311++G(3d,3p). Os cálculos de otimização de geometria, de constantes de força quadráticas e de cargas e dipolos atômicos ChelpG foram realizados pelo programa de estrutura eletrônica Gaussian $03,{ }^{3}$ enquanto as cargas e dipolos atômicos QTAIM foram calculadas pelo programa para análise topológica da densidade eletrônica Morphy 98. ${ }^{28}$ No cálculo das cargas e dipolos atômicos ChelpG, duas restrições foram impostas: a soma das cargas atômicas deve reproduzir a carga total do sistema e a soma dos dipolos atômicos deve reproduzir o vetor momento dipolar elétrico do sistema. As derivadas numéricas das cargas e dos dipolos atômicos foram calculadas com um deslocamento atômico igual a 0,01 A. A simetria molecular foi explorada pelo programa Placzek no cálculo destas derivadas, reduzindo o número de cálculos em geometrias fora do equilíbrio, respectivamente para $\mathrm{CH}_{4}, \mathrm{NH}_{3}$ e $\mathrm{H}_{2} \mathrm{O}$, de 30, 24 e 18 para 12, diminuindo significativamente o esforço computacional demandado no cálculo das intensidades infravermelhas CCFDF. As intensidades não CCFDF, nível MP2/6-311++G(3d,3p), foram calculadas pelo programa Gaussian, que obtêm analiticamente as derivadas geométricas do momento dipolar elétrico molecular a partir da densidade eletrônica MP2.

\section{RESULTADOS E DISCUSSÃO}

O primeiro aspecto que deve ser levado em consideração na avaliação da implementação do modelo CCFDF diz respeito à concordância observada entre as intensidades obtidas diretamente da densidade eletrônica do sistema e aquelas oriundas da soma das parcelas de carga, fluxo de carga e fluxo de dipolo. Seus valores, para água, amônia e metano, são apresentados na Tabela 1, juntamente com os valores obtidos através do modelo CCFDF com cargas e dipolos atômicos ChelpG e também QTAIM. Os valores das intensidades observados experimentalmente também estão listados nesta tabela. As diferenças que podem ocorrer entre as intensidades CCFDF/QTAIM e as obtidas diretamente da função de onda do sistema têm sua origem em imprecisões introduzidas por determinação numérica das superfícies de fluxo zero, as quais definem as fronteiras dos átomos na molécula; integração numérica dentro dos limites definidos na etapa anterior e, método numérico utilizado para calcular as derivadas das cargas e dos dipolos atômicos (aproximação linear e tamanho do deslocamento). Em se tratando do modelo ChelpG, em razão das restrições impostas ao cálculo das cargas e dos dipolos atômicos, tais diferenças surgem essencialmente do método numérico de derivação mencionado anteriormente. Para avaliar este tipo de limitação da implementação CCFDF apresentada neste trabalho, os erros RMS para os modelos CCFDF/ChelpG e CCFDF/QTAIM foram calculados, tomando como referência os dados MP2 analíticos. A concordância observada é excelente, onde o modelo CCFDF/ChelpG mostra um erro igual a $3,4 \mathrm{~km} / \mathrm{mol}$, enquanto o CCFDF com cargas QTAIM apresenta um erro ainda menor, de $2,1 \mathrm{~km} / \mathrm{mol}$. Observando os valores das intensidades na Tabela 1 , conclui-se que a maior parcela para este desvio vem da transição relativa ao modo $v_{2}\left(a_{1}\right)$ da amônia (deformação de ângulo simétrica), cujos valores CCFDF superestimam o respectivo valor MP2 em cerca de 5 a $10 \mathrm{~km} / \mathrm{moI}$, dependendo do modelo de cargas e dipolos atômicos considerado. A concordância entre as intensidades calculadas e os respectivos valores observados, expressa pelo erro RMS, tomando como referência os dados experimentais, é, respectivamente, de 13,0, 14,7 e 13,3 km/mol para os modelos MP2, CCFDF/ChelpG e CCFDF/ QTAIM. A dispersão entre estes valores de intensidade calculados e experimentais é ilustrada na Figura 1.

Na Tabela 2 são mostradas as contribuições de carga, fluxo de carga e fluxo de dipolo para a derivada do momento dipolar molecular com respeito a cada vibração normal de água, amônia e metano, cuja 
Tabela 1. Intensidades de absorção no infravermelho (em km/mol) para as bandas fundamentais da água, amônia e metano. Valores observados experimentalmente e calculados segundo os modelos MP2/6-311++G(3d,3p), CCFDF/ChelpG/MP2/6-311++G(3d,3p) e CCFDF/QTAIM/ MP2/6-311++G(3d,3p)

\begin{tabular}{|c|c|c|c|c|c|c|}
\hline Molécula & Modo & $\tilde{\mathrm{v}}\left(\mathrm{cm}^{-1}\right)^{a}$ & $A_{k}(\exp )^{b}$ & $A_{k}(M P 2)$ & $A_{k}($ Chelp $G)$ & $A_{k}(Q T A I M)$ \\
\hline \multirow{3}{*}{$\mathrm{H}_{2} \mathrm{O}$} & $v_{1}\left(a_{1}\right)$ & 3657 & 2,93 & 5,5 & 5,5 & 5,5 \\
\hline & $v_{2}\left(a_{1}\right)$ & 1595 & 62,5 & 68,5 & 68,4 & 67,2 \\
\hline & $v_{3}\left(b_{2}\right)$ & 3756 & 41,7 & 70,8 & 70,8 & 70,1 \\
\hline \multirow{4}{*}{$\mathrm{NH}_{3}$} & $v_{1}\left(a_{1}\right)$ & 3337 & 7,0 & 3,6 & 3,1 & 3,0 \\
\hline & $v_{2}\left(a_{1}\right)$ & 950 & 131,0 & 147,8 & 157,9 & 152,4 \\
\hline & $v_{3}(e)$ & 3444 & 3,3 & 14,6 & 14,3 & 12,9 \\
\hline & $v_{4}(e)$ & 1627 & 27,7 & 29,0 & 27,4 & 32,3 \\
\hline \multirow{2}{*}{$\mathrm{CH}_{4}$} & $v_{3}\left(f_{2}\right)$ & 3019 & 68,8 & 54,4 & 54,9 & 55,6 \\
\hline & $v_{4}\left(f_{2}\right)$ & 1306 & 34,2 & 30,8 & 30,3 & 32,4 \\
\hline
\end{tabular}

${ }^{a}$ Frequiências vibracionais experimentais compiladas por Shimanouchi ${ }^{30} .{ }^{b}$ As intensidades experimentais para água foram extraídas da ref. 31 . As intensidades experimentais apresentadas para a amônia são a média das intensidades relatadas nas refs. 32 e 33 e, para o metano, a média das intensidades reportadas nas refs. 34 e 36

Tabela 2. Contribuições de carga (C), fluxo de carga (FC) e fluxo de dipolo (FD) para as derivadas do momento dipolar em relação às coordenadas normais, para água, amônia e metano, em unidades de elétron (e), obtidas pelos modelos CCFDF/ChelpG e CCFDF/QTAIM no nível de teoria MP2/6-311++G(3d,3p)

\begin{tabular}{|c|c|c|c|c|c|c|c|}
\hline Molécula & Modo & $\tilde{v}\left(\mathrm{~cm}^{-1}\right)$ & Modelo & $\mathrm{C}$ & $\mathrm{FC}$ & FD & Total \\
\hline \multirow{6}{*}{$\mathrm{H}_{2} \mathrm{O}$} & \multirow{2}{*}{$v_{1}\left(a_{1}\right)$} & \multirow{2}{*}{3657} & ChelpG & $-0,72$ & $+1,04$ & $-0,39$ & $-0,07$ \\
\hline & & & QTAIM & $-0,52$ & $+0,62$ & $-0,18$ & $-0,08$ \\
\hline & \multirow[b]{2}{*}{$v_{2}\left(a_{1}\right)$} & \multirow{2}{*}{1595} & ChelpG & $+1,02$ & $-0,03$ & $-0,71$ & $+0,28$ \\
\hline & & & QTAIM & $+0,73$ & $-0,23$ & $-0,23$ & $+0,27$ \\
\hline & \multirow{2}{*}{$v_{3}\left(b_{2}\right)$} & \multirow{2}{*}{3756} & ChelpG & $+1,02$ & $-1,45$ & $+0,71$ & $+0,28$ \\
\hline & & & QTAIM & $+0,73$ & $-0,78$ & $+0,33$ & $+0,28$ \\
\hline \multirow{8}{*}{$\mathrm{NH}_{3}$} & \multirow{2}{*}{$v_{1}\left(a_{1}\right)$} & \multirow{2}{*}{3337} & ChelpG & $-0,45$ & $+2,32$ & $-1,81$ & $+0,06$ \\
\hline & & & QTAIM & $-0,21$ & $+0,03$ & $+0,24$ & $+0,06$ \\
\hline & \multirow{2}{*}{$v_{2}\left(a_{1}\right)$} & \multirow{2}{*}{950} & ChelpG & $+1,39$ & $-3,79$ & $+2,84$ & $+0,44$ \\
\hline & & & QTAIM & $+0,64$ & $-0,14$ & $-0,07$ & $+0,43$ \\
\hline & \multirow{2}{*}{$v_{3}(e)$} & \multirow{2}{*}{3444} & ChelpG & $+0,95$ & $-1,70$ & $+0,84$ & $+0,09$ \\
\hline & & & QTAIM & $+0,44$ & $-0,11$ & $-0,24$ & $+0,09$ \\
\hline & \multirow{2}{*}{$v_{4}(e)$} & \multirow{2}{*}{1627} & ChelpG & $+0,81$ & $+1,03$ & $-1,71$ & $+0,13$ \\
\hline & & & QTAIM & $+0,37$ & $-0,18$ & $-0,06$ & $+0,13$ \\
\hline \multirow{4}{*}{$\mathrm{CH}_{4}$} & \multirow{2}{*}{$v_{3}\left(f_{2}\right)$} & \multirow{2}{*}{3019} & ChelpG & $-0,45$ & $+0,99$ & $-0,68$ & $-0,14$ \\
\hline & & & QTAIM & $-0,03$ & $+0,66$ & $-0,77$ & $-0,14$ \\
\hline & \multirow{2}{*}{$v_{4}\left(f_{2}\right)$} & \multirow{2}{*}{1306} & ChelpG & $-0,62$ & $-1,23$ & $+1,96$ & $+0,11$ \\
\hline & & & QTAIM & $-0,04$ & $-0,14$ & $+0,30$ & $+0,12$ \\
\hline
\end{tabular}

transição fundamental é ativa no infravermelho. A decomposição CCFDF apresentada nesta tabela foi calculada com os modelos de cargas e dipolos atômicos ChelpG e QTAIM. Os valores contidos nesta tabela são a norma dos vetores $\mathbf{C}_{\mathrm{Q}_{\mathrm{k}}}, \mathbf{F C}_{\mathrm{Q}_{\mathrm{k}}}$ e $\mathbf{F D}_{\mathrm{Q}_{\mathrm{k}}}$ definidos na Equação 14, mais um respectivo sinal, + ou -. Para atribuir este sinal, tornou-se o sinal da maior, em módulo, das três componentes do respectivo vetor de carga ou fluxo, isto porque, com frequiência, existe uma componente majoritária nestes vetores. Por exemplo, as

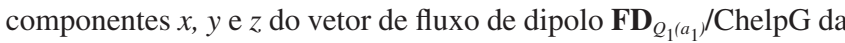
água são, respectivamente, 0,$00 ; 0,00$ e $-0,39 e$, possuindo, portanto, norma igual a $\mathrm{O}, 39$ e um sinal negativo. A análise destes termos com o respectivo sinal permite avaliar não somente a magnitude de cada urna das três componentes CCFDF para uma dada vibração, mas também se a mesma cresce ou diminui ao longo do movimento de vibração. A observação dos valores contidos nesta tabela mostra que ambos os modelos prevêem intensidades infravermelhas muito similares. No entanto, a norma e o sentido dos termos de carga, flu- xo de carga e fluxo de dipolo são bastante dependentes do modelo usado no cálculo das cargas e dos dipolos atômicos, podendo variar significativamente, em razão das características teóricas de cada um destes modelos.

\section{CONCLUSÕES}

Neste artigo é apresentada a primeira implementação computacional capaz de automatizar todos os procedimentos envolvidos no cálculo das intensidades no espectro infravermelho segundo o modelo Carga-Fluxo de Carga-Fluxo de Dipolo. Esta implementação foi efetuada no programa Placzek, escrito em linguagem Fortran77, desenvolvido para o cálculo de intensidades infravermelhas e Raman em diversos níveis de teoria ab initio e também DFT. Na forma como o modelo CCFDF foi implementado, o elevado número de tarefas requeridas em um cálculo CCFDF é executado de forma totalmente automática e eficaz, pois este programa é capaz de explorar a sime- 


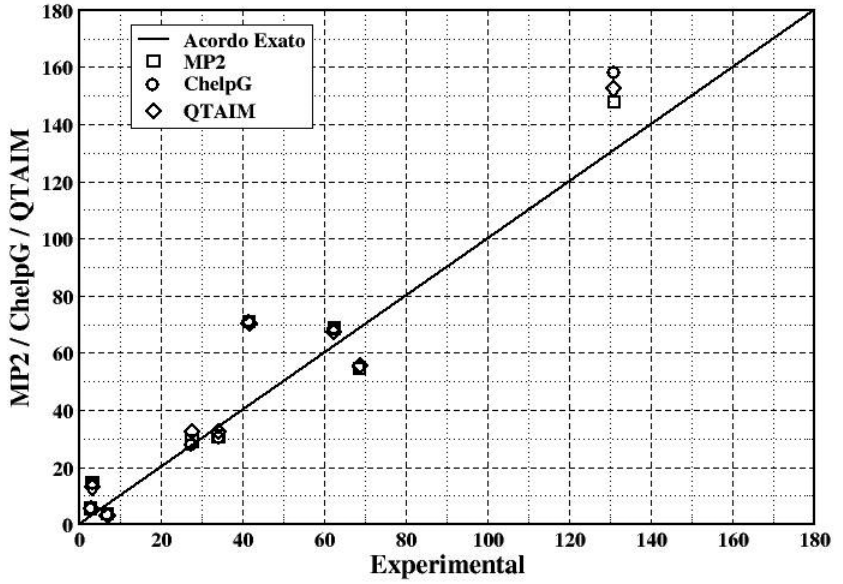

Figura 1. Intensidades infraverrnelhas (em $\mathrm{km} / \mathrm{mol}$ ) obtidas diretamente da densidade eletrônica MP2/6-311++G(3d,3p) e calculadas com cargas Chelp $G$ e QTAIM usando o modelo CCFDF plotadas contra os valores experimentais. Dados relativos às transições fundamentais de água, amônia e metano

tria molecular, evitando cálculos redundantes e, por conseqüência, reduzindo o esforço computacional exigido na análise CCFDF. Com o programa Placzek, as intensidades e seus respectivos termos de carga e de fluxo podem ser obtidos nos níveis de teoria Hartree-Fock, MP2, Multiconfiguracionais (como CASSCF ou QCISD) e DFT. Com relação aos modelos para cálculo de cargas e de dipolos atômicos, as implementações QTAIM nos programas Gaussian ou Morphy podem ser usadas com o programa Placzek, além da implementação ChelpG no programa Gaussian. Cálculos ilustrativos inéditos foram realizados com o programa Placzek para as moléculas de água, amônia e metano, no nível de teoria MP2/6-311++G(3d,3p), utilizando tanto o modelo ChelpG como o QTAIM/Morphy. Estes resultados são comparados com os obtidos diretamente da densidade eletrônica MP2/6$311++\mathrm{G}(3 \mathrm{~d}, 3 \mathrm{p})$, calculados com o programa Gaussian, mostrando uma excelente concordância entre as intensidades infravermelhas para ambos os modelos ChelpG e QTAIM.

\section{AGRADECIMENTOS}

À Fundação de Amparo à Pesquisa do Estado de São Paulo (Fapesp) pelo apoio financeiro (projeto $\mathrm{n}^{\circ} 06 / 53260-6$ ). J. V. da Silva Jr. agradece à Fapesp. T. C. F. Gomes e L. N. Vidal agradecem ao CNPq pelas bolsas de pós-graduação.

\section{REFERÊNCIAS}

1. Seidler, P.; Kongsted, J.; Christiansen, O.; J. Phys. Chern. A 2007, 111, 11205.

2. Davics, M., ed.; Infra-Red Spectroscopy and Molecular Structure, Elsevier Publishing Company: Amsterdam, 1963.

3. Frisch, M. J.; Trucks, G.. W.; Schlegel, H. B.; Scuseria, G. E.; Robb, M. A.; Cheeseman, J. R.; Montgomery, Jr., J. A.; Vreven, T.; Kudin, K. N.; Burant, J. C.; Millam, J. M.; Iyengar, S. S.; Tomasi, J.; Barone, V.; Mennucci, B.; Cossi, M.; Scalmani, G.; Rega, N.; Petersson, G. A.; Nakatsuji, H.; Hada, M.; Ehara, M.; Toyota, K.; Fukuda, R.; Hasegawa, J.; Ishida, M.; Nakajima, T.; Honda, Y.; Kitao, O.; Nakai, H.; Klene, M.; Li, X.; Knox, J. E.; Hratchian, H. P.; Cross, J. B.; Bakken, V.; Adamo, C.; Jaramillo, J.; Gomperts, R.; Stratmann, R. E.; Yazyev, O.; Austin, A. J.; Cammi, R.; Pomelli, C.; Ochterski, J. W.; Ayala, P. Y.; Morokuma, K.; Voth, G. A.; Salvador, P.; Dannenberg, J. J.; Zakrzewski, V. G.; Dapprich, S.; Daniels, A. D.; Strain, M. C.; Farkas, O.; Malick, D. K.;
Rabuck, A. D.; Raghavachari, K.; Foresman, J. B.; Ortiz, J. V.; Cui, Q.; Baboul, A. G.; Clifford, S.; Cioslowski, J.; Stefanov, B. B.; Liu, G.; Liashenko, A.; Piskorz, P.; Komaromi, I.; Martin, R. L.; Fox, D. J.; Keith, T.; Al-Laham, M. A.; Peng, C. Y.; Nanayakkara, A.; Challacombe, M.; Gill, P. M. W.; Johnson, B.; Chen, W.; Wong, M. W.; Gonzalez, C.; Pople, J. A.; Gaussian 03, Revision D.02, Gaussian, Inc., Wallingford CT, 2004

4. Sehmidt, M. W.; Baldridge, K. K.; Boatz, J. A.; Elbert, S. T.; Gordon, M. S.; Jensen, J. H.; Koseki, S.; Matsunaga, N.; Nguyen, K. A.; Su, S.; Windus, T. L.; Dupuis, M.; Montgomery, J. A.; J. Comput. Chem. 1993, 14, 1347.

5. Dalton, a molecular electronic structure program, Release 2.0 (2005), see http://www.kjemi.uio.no/software/dalton/dalton.html, acessada em Abril 2006.

6. Velde, G. T.; Bickelhaupt, F. M.; Baerends, E. J.; Guerra, C. F.; Gisbergen, S. .J.; Snijders, J. G.; Ziegler, T.; J. Comput. Chem. 2001, 22, 931.

7. da Silva Jr., J. V.; Haiduke, R. L. A.; Bruns, R. E.; J. Phys. Chem. A 2006, $110,4839$.

8. King, W. T.; Mast, G.. B.; J. Phys. Chem. 1976, 80, 2521.

9. Ferreira, M. M. C.; J. Phys. Chem. 1990, 94, 3220.

10. Choi, C. H.; Kertesz, M.; Chem. Phys. Lett. 1996, 263, 697.

11. Mullikcn, R. S.; J. Chem. Phys. 1955, 23, 1833.

12. Mulliken, R. S.; J. Chem. Phys. 1955, 23, 1841.

13. Mulliken, R. S.; J. Chem. Phys. 1962, 36, 3428.

14. Ciolowski, J.; J. Am. Chem. Soc. 1989, 111, 8333.

15. Haiduke, R. L. A.; Bruns, R. E.; J. Phys. Chem. A 2005, 109, 2680.

16. Bader, R. F. W.; Larouche, A.; Gatti, C.; Carroll, M. T.; MacDougall, P. J.; J. Chem. Phys. 1987, 87, 1142.

17. Haiduke, R. L. A.; de Oliveira, A. E.; Bruns, R. E.; J. Phys. Chem. A 2004, 108, 6788.

18. Bader, R. F. W. Atoms in Molecules: a Quantum Theory; Claredon Press: Oxford, 1990.

19. Bader, R. F. W.; Acc. Chem. Res. 1985, 18, 9.

20. Faria, S. H. D.; da Silva Jr., J. V.; Haiduke, R. L. A.; Vidal, L. N.; Vazquez, P. A. M.; Bruns, R. E.; J. Phys. Chem. A 2007, 111, 7870.

21. Vidal, L. N.; Vazquez, P. A. M.; Quim. Nova 2003, 26, 507.

22. Vidal, L. N.; Vazquez, P. A. M.; Int. J. Quantum Chem. 2005, 103, 632.

23. Breneman, C. M.; Wiberg, K. B.; J. Comput. Chem. 1990, 11, 361.

24. Chirlian, L. E.; Francl, M. M.; J. Comput. Chem. 1987, 8, 894.

25. Person, W. B.; Newton, J. H.; J. Chem. Phys. 1974, 61, 1040.

26. Biarge, J. F.; Morcillo, J.; Herranz, J.; Anales de la Real Sociedad Espanola de Fisica y Química 1961, 57A, 81

27. Hamming, R. W. Numerical Methods for Scientists and Engineers; Dover Publications, Inc.: New York, 1986.

28. MORPHY98, a program written by P. L. A. Popelier with a contribution from R. G. A. Bone; UMIST, Manchester, England, EU (1998).

29. Wilson Jr., E. B.; Decius, J. C.; Cross, P. C.; Molecular Vibrations, The Theory of Infrared and Raman Vibrational Spectra, Dover Publications, Inc.: New York, 1980.

30. Shimanouchi, T., Molecular Vibrational Frequencies, Em NIST Chemistry WebBook; NIST Standard Reference Database 69; National Institute of Standards and Technology: Gaithersburg MD, 20899, 2001, http:// webbook.nist.gov, acessada em Abril 2008.

31. Bishop, D. M.; Cheung, L. M.; J. Phys. Chem. Ref. Data 1982, 11, 119.

32. McKean, D. C.; Schatz, P. N.; J. Chem. Phys. 1956, 24, 316

33. Koops, T.; Visser, T.; Smit, W. M. A.; J. Mol. Struct. 1983, 96, 203.

34. Bodc, J. H. G.; Smit, W. M. A.; J. Phys. Chem. 1980, 84, 198.

35. Saëki, S.; Mizuno, S.; Kondo, S.; Spectrochim. Acta, Part A 1976, 32, 403.

36. Heicklen, J.; Spectrochim. Acta, Part A 1961, 17, 201. 